\title{
Anatomia comparada do lenho de Caryocar brasiliense (Caryocaraceae) em fisionomias de cerradão e cerrado sensu stricto ${ }^{1}$
}

\author{
Comparative wood anatomy of Caryocar brasiliense Camb. (Caryocaraceae) \\ occurring in "cerradão" and "cerrado" sensu stricto formations
}

\author{
Alessandra Regina Aguilar Voigt ${ }^{2,5}$, Patricia Soffiatti ${ }^{2}$, Mário Tomazello Filho ${ }^{3}$, \\ Claudio Sergio Lisi ${ }^{4} \&$ Maria Regina Torres Boeger ${ }^{2}$
}

\begin{abstract}
Resumo
Este trabalho verificou a influência das condições ambientais na estrutura do xilema secundário da espécie Caryocar brasiliense Camb. (pequizeiro) através de um estudo anatômico comparado do lenho de árvores procedentes de fisionomias de cerradão e cerrado s.s., no município de Santa Rita do Passa Quatro, SP. As características do lenho foram analisadas qualitativa e quantitativamente, sendo as últimas comparadas pela análise de variância, bem como por análises multivariadas de agrupamentos e componentes principais. A análise de variância demonstrou existir diferenças significativas para oito das dezesseis variáveis analisadas, bem como uma grande variação entre árvores da mesma localidade, aspecto corroborado pela análise de agrupamentos. A matriz de correlação entre variáveis indicou, de modo geral, relações inversas entre dimensões longitudinais e radiais dos elementos anatômicos. Pode-se inferir pela variabilidade significativa entre árvores que a diferenciação das fisionomias ocorre de forma gradual, não se observando um conjunto de características anatômicas suficientemente marcantes que tipifiquem as fisionomias estudadas.
\end{abstract}

Palavras-chave: anatomia ecológica, pequi, tendências ecológicas, xilema secundário.

\begin{abstract}
This study aimed to verify the influence of ambient conditions on the structure of secondary xylem of Caryocar brasiliense Camb. ("pequizeiro") through a comparative anatomical study of two populations belonging to "cerradão" and "cerrado s. s." (Brazilian savannas), in the city of Santa Rita do Passa Quatro, São Paulo. The wood was described qualitatively, and quantitative data were analyzed using analysis of variance, as well as multivariate and principal components analysis. The analysis of variance showed significant differences for eight out of sixteen anatomical parameters, as well as great variation between trees of the same locality, an aspect corroborated by multivariate analysis. The correlation matrix showed generally inverse correlations between longitudinal and radial measurements of the anatomical characters. Based on the significant variability found between trees we can infer that the differentiation of the formations occurs gradually, since a marked set of anatomical characters that would individualize each type of vegetation was not observed.
\end{abstract}

Key words: ecological anatomy, "pequi", ecological trends, secondary xylem.

\section{Introdução}

O Cerrado, segundo maior bioma brasileiro, é composto por um mosaico de fitofisionomias que abrangem formações florestais (mata ciliar, mata de galeria, mata seca e cerradão), savânicas (cerrado denso, típico, ralo, rupestre, vereda, parque de cerrado e palmeiral) e campestres (campos sujo, limpo e rupestre) (Ribeiro \& Walter 1998). A ocorrência dessas fisionomias pode ser determinada por manchas de solos mais ou menos pobres, pela

\footnotetext{
${ }^{1}$ Parte da dissertação de Mestrado da primeira autora. Programa de Pós-graduação em Botânica, Universidade Federal do Paraná, C.P. 19031, 81531-990, Curitiba, PR. ${ }^{2}$ Universidade Federal do Paraná, Depto. Botânica, C.P. 19031, 81531-990, Curitiba, PR.

${ }^{3}$ Universidade de São Paulo, Escola Superior de Agricultura “Luiz de Queiroz”, Depto. Ciências Florestais, Av. Pádua Dias 11, C.P. 09, 13418-900, Piracicaba, SP.

${ }^{4}$ Universidade Federal de Sergipe, Depto. Biologia, Av. Marechal Rondon s/n, 49100-000, São Cristóvão, SE.

54Ator para correspondência: arvoigt@gmail.com
} 
irregularidade dos regimes pluviométricos, queimadas características de cada local e pela ação humana (Coutinho 2008). Os primeiros estudos que relacionaram as características dos solos com a vegetação do Cerrado datam da década de 1950 (Alvim \& Araújo 1952). Anteriormente a esse período, alguns trabalhos já indicavam que as características xeromórficas das plantas de cerrado não se relacionavam exclusivamente com a disponibilidade hídrica (Rawitscher \& Ferri 1943), contrariando o afirmado por Warming (1908), de que a escassez de água constituía o fator limitante para a vegetação.

Após revisar extensamente trabalhos clássicos sobre o Cerrado, Rizzini (1976) alerta para a existência de uma grande variação de respostas das plantas às oscilações na disponibilidade hídrica, abrangendo desde espécies que aparentemente não diminuíam a transpiração até espécies que apresentavam uma restrição considerável desta durante a estação seca. Estudos demonstraram que a grande maioria das espécies lenhosas restringe a abertura estomática durante a estação seca (Perez \& Moraes 1991; Franco \& Lüttge 2002) e que algumas espécies apresentam restrição estomática mesmo durante a estação chuvosa, dependendo da demanda evaporativa da atmosfera (Naves-Barbiero et al. 2000; Franco \& Lüttge 2002). Nas diferenciações fisionômicas, a diminuição da biomassa e a modificação da composição florística também demonstram possuir correlação com a disponibilidade de nutrientes e o aumento da saturação de alumínio no solo. A maior dificuldade das plantas em sintetizar proteínas, sob tais características limitantes do solo, levariam a uma produção excessiva de carboidratos e gorduras. Tal fato justificaria o pequeno porte das árvores e a abundância de características esclerenquimáticas, justamente os aspectos considerados xeromórficos (Arens $1958 \mathrm{a}, \mathrm{b}$ ).

Neste panorama, os estudos de anatomia ecológica do xilema secundário podem fornecer dados importantes para uma maior compreensão das interações entre as condições ambientais e o desenvolvimento das plantas em ambientes de cerrado. Estudos demonstram que muito da diversidade estrutural encontrada neste tecido possui uma explicação funcional e adaptativa, podendo ser diretamente relacionada ao hábito das plantas e às condições ambientais (Dickison 2000), uma vez que fatores como seca, inundação, altitude, latitude, constituição e fertilidade do solo, estádios sucessionais da vegetação e poluição podem alterar significativamente sua estrutura anatômica (Baas 1973; Carlquist \& Hoekman 1985; Baas \& Schweingruber 1987). As relações entre a estrutura da madeira e as características climáticas têm sido amplamente estudadas, sendo Baas (1973) e Carlquist (1975) os pioneiros nesta abordagem. As espécies tropicais têm recebido crescente atenção no Brasil, contudo, alguns trabalhos mostram resultados controversos com relação às grandes tendências estabelecidas para as floras mundiais, o que reforça a necessidade de se ampliar este tipo de estudo no Brasil.

O pequizeiro (Caryocar brasiliense Camb.), uma das poucas espécies com ampla distribuição no bioma Cerrado (Ratter et al. 1996), é uma planta decídua presente em várias fisionomias, com distribuição em doze Estados e no Distrito Federal (Silva Júnior 2005). Pelo fato de ser pouco exigente quanto à fertilidade do solo (Haridasan 2005), os elevados teores minerais de seus frutos chegam a surpreender pela sua alta concentração nutricional, conduzindo à hipótese de que se trata de uma planta bastante eficiente na extração de nutrientes. Com isso, o estudo anatômico do xilema secundário desta espécie pode fornecer dados importantes para a compreensão de algumas relações entre as plantas e variáveis ambientais em áreas de cerrado. Neste panorama, tendo-se em vista a importância deste bioma e a necessidade de se ampliar o número de pesquisas referentes à anatomia ecológica do xilema secundário de espécies brasileiras, este trabalho traz um estudo anatômico comparado de duas populações de Caryocar brasiliense em fisionomias de cerradão e cerrado s.s., com o objetivo de verificar tendências ecológicas da espécie nestes ambientes.

\section{Material e Métodos}

\section{Caracterização da área de estudo}

O estudo foi realizado na gleba "Pé-degigante", parte integrante do Parque Estadual da Vassununga, localizada no município de Santa Rita do Passa Quatro (SP). Pertencente ao Instituto Florestal do Estado de São Paulo, a gleba ocupa uma área de 1.225 ha com coordenadas centrais $21^{\circ} 37^{\prime} 30^{\prime \prime}$ 'S e $47^{\circ} 37^{\prime} 30^{\prime}$ ' $\mathrm{W}$, sendo constituída predominantemente por vegetação de cerrado em suas diversas fisionomias, e em menor parte por manchas de floresta estacional semidecídua (Ruggiero et al. 2006). O presente trabalho foi realizado em duas áreas da referida gleba, sendo uma em fisionomia de cerradão e outra em cerrado s.s. (Fig. 1). As áreas do Parque encontram-se entre 
altitudes de 600 - 780 m, com tipo climático Cwa (temperado macrotérmico, moderadamente chuvoso, de inverno seco não rigoroso) segundo classificação de Köppen (1948). A precipitação média anual é de $1.478 \mathrm{~mm}$, com média de $20 \mathrm{~mm}$ no mês mais seco (julho) e $282 \mathrm{~mm}$ no mês de maior precipitação (janeiro) (Fig. 2). Apresenta déficit hídrico de 6 meses (abril a setembro, com pico em agosto) e excedente de 5 meses (novembro a março, com pico em janeiro). A temperatura média anual é de $21,1^{\circ} \mathrm{C}$, com mínima de $17,6^{\circ} \mathrm{C}$ em julho e máxima de $23,5^{\circ} \mathrm{C}$ em fevereiro (Rolim \& Sentelhas 2008), podendo ocorrer geadas durante o inverno (junho a agosto) (Ruggiero et al. 2006).

Nas áreas mais altas sobre neossolos quartzarênicos ocorrem fisionomias de cerrado que têm maior participação do componente arbóreo, tais como cerrado s.s., cerrado com estrato herbáceo ralo e cerradão (Ruggiero et al. 2006). As diferentes fisionomias de cerrado s.s. não demonstram ter correlação clara com as classes de solo, tampouco com a fertilidade (Ruggiero et al. 2002, 2006), o que pode indicar que sejam determinadas por uma combinação mais complexa de fatores, na qual a variação de água subterrânea seja de grande importância (Ruggiero et al. 2006).

Levantamentos pedológicos previamente realizados em toda a gleba "Pé-de-gigante" por
Ruggiero et al. (2006) e Ruggiero \& Pivello (2005) serviram de base para a construção de gráficos indicativos do perfil da fertilidade dos solos (Fig. 3), sendo os mesmos específicos para as áreas estudadas.

\section{Coleta de material e preparo das amostras}

Para o estudo anatômico do lenho da espécie Caryocar brasiliense empregou-se o método de coleta não destrutivo (extrator motorizado). Foram coletadas amostras cilíndricas (2,0 cm de diâmetro e comprimento variável) de seis árvores em área de cerradão e seis árvores em cerrado s.s., sendo estas obtidas em regiões do tronco livres de tortuosidades e, sempre que possível, à altura do $\operatorname{DAP}(1,30 \mathrm{~m})$. As árvores possuíam dimensões de 13,1 - 24,5 cm de diâmetro em área de cerradão e 18,1 - 24,5 cm em cerrado s.s. Os corpos de prova, com volume aproximado de $1,0 \mathrm{~cm}^{3}(1,0 \times 1,0 \times 1,0 \mathrm{~cm})$ foram obtidos a partir da porção mais externa do tronco, distando aproximadamente $1 \mathrm{~cm}$ da região do câmbio vascular. As amostras foram preparadas de acordo com métodos usualmente empregados em estudos anatômicos do xilema secundário, segundo as normas COPANT (1974), IAWA Committee (1989) e IBAMA (1992). As lâminas utilizadas no estudo encontram-se depositadas nos Laboratórios de Anatomia e Identificação de Madeiras dos

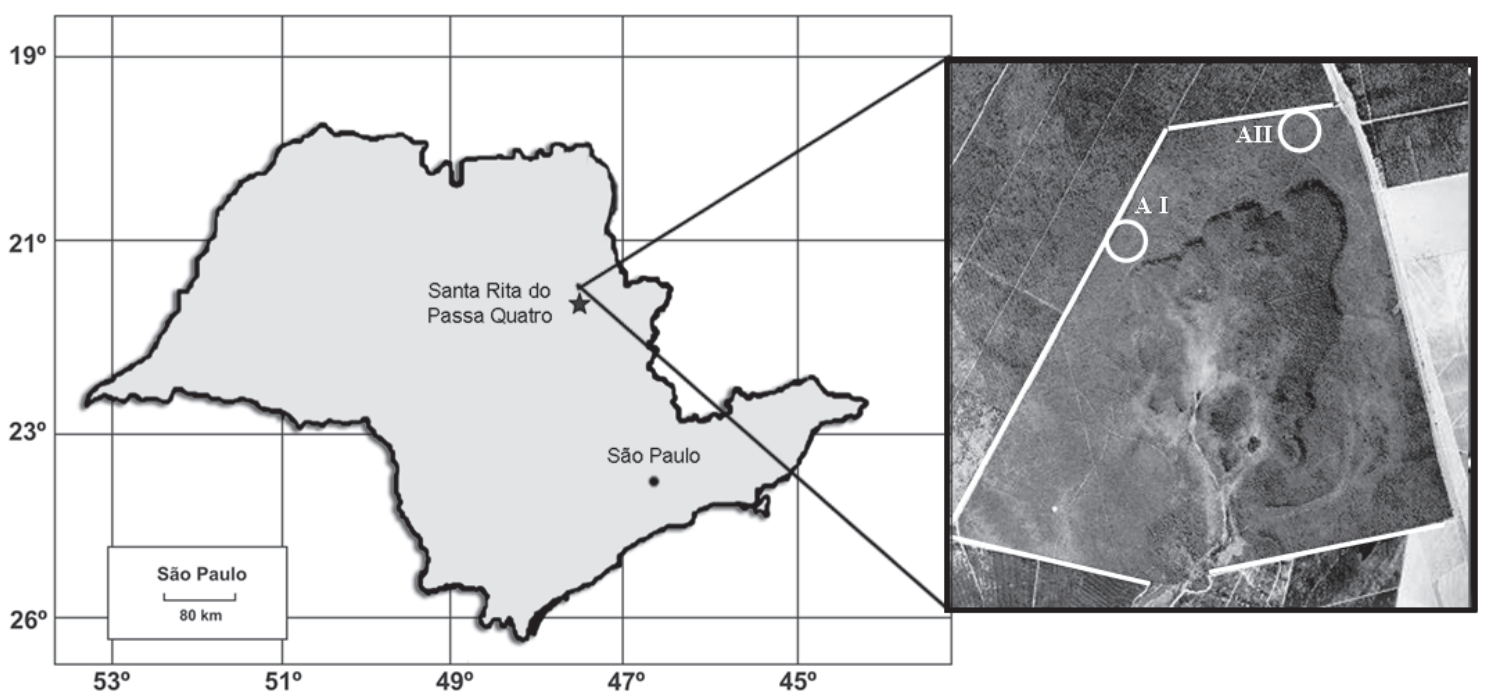

Figura 1 - Localização das áreas de estudo na gleba Pé-de-gigante do Parque Estadual da Vassununga, município de Santa Rita do Passa Quatro, SP: (AI) cerradão; (AII) cerrado sensu stricto.

Figure 1 - Location of the study sites in "Pé-de-gigante" of Parque Estadual da Vassununga, Santa Rira do Passa Quatro town, SP: (AI) "cerradão"; (AII) "cerrado" sensu stricto. 


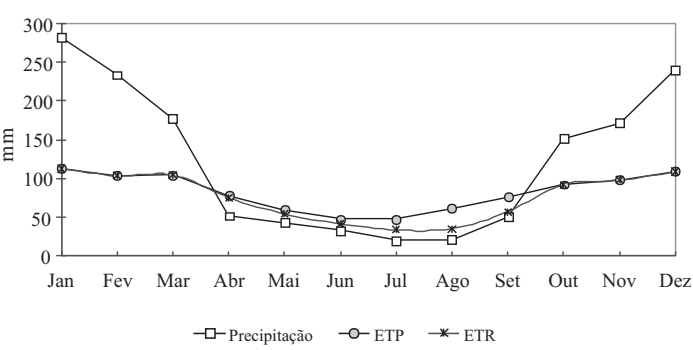

Figura 2 - Precipitação e balanço hídrico mensal para a região de Santa Rita do Passa Quatro, SP. ETP: Evapotranspiração potencial; ETR: Evapotranspiração real. (Rolim \& Sentelhas 2008).

Figure 2 - Monthly rainfall and hydric balance for the region of Santa Rita do Passa Quatro, SP. ETP: Potential evapotranspiration; ETR: Real evapotranspiration (Rolim \& Sentelhas 2008).

Departamentos de Ciências Florestais da Universidade de São Paulo (ESALQ/USP) e Universidade Federal do Paraná (UFPR). Utilizouse de material macerado e cortes histológicos para a análise de dezesseis variáveis, sendo três relativas às fibras (comprimento, diâmetro total e espessura da parede celular), três aos raios parenquimáticos (altura, largura e frequência) e dez aos elementos de vaso (agrupamento: porcentagem de vasos solitários, múltiplos de 2, 3, 4 e 5 a mais células; diâmetro, comprimento, frequência, área e porcentagem). O número de medições por árvore foi fixado em (i) $n=50$ elementos para as variáveis referentes às suas dimensões; $\mathrm{e}$ (ii) $\mathrm{n}=5$ imagens de $6,58 \mathrm{~mm}^{2}$ para as variáveis dependentes de área, mensurando-se, deste modo, um mínimo de $33 \%$ da área de cada corpo de prova. Com o objetivo de verificar possíveis correlações entre estrutura anatômica do xilema e características dos solos foram utilizados dados de levantamentos pedológicos previamente realizados no local por Ruggiero \& Pivello (2005) e Ruggiero et al. (2006).

\section{Análise estatística}

Aplicou-se teste de Bartlett (Steel et al. 1997) com o objetivo de verificar a homogeneidade das variâncias, sendo as variáveis não homogêneas devidamente transformadas. Empregou-se análise de variância em delineamento hierárquico, avaliando-se áreas e árvores dentro de áreas. As médias foram comparadas mediante aplicação do teste $\mathrm{F}(\mathrm{p}<0,05)$ para verificar se as diferenças podem ser consideradas significativas do ponto de vista estatístico. De maneira análoga, aplicouse o teste de Tukey $(\mathrm{p}<0,05)$ para verificar em quais fatores residem tais diferenças. Os valores médios por árvore foram empregados em análises multivariadas de agrupamento ("cluster") e componentes principais "biplot” (PCA).

\section{Resultados e Discussão}

\section{Caracterização anatômica do lenho}

Os dados qualitativos obtidos no presente estudo para a espécie Caryocar brasiliense assemelham-se aos relatados na literatura por Mello (1970) e Araújo \& Mattos (1973). O xilema secundário é caracterizado pela porosidade difusa, vasos solitários e múltiplos radiais de 2-3 células (Fig. 4a-b), fibras libriformes, parênquima axial predominantemente apotraqueal difuso a difuso em agregados, às vezes em faixas estreitas, tendendo a reticulado; pontoações intervasculares alternas de contorno poligonal a oval, em pares areolados, placas de perfuração simples; pontoações parênquimo-vasculares semi-areoladas, às vezes simplificadas e escalariformes, dispostas irregularmente em forma e arranjo (Fig. 4c-d), pontoações radiovasculares semelhantes às parenquimovasculares (Fig. 4f); camadas de crescimento distintas a pouco distintas (Fig. 4a-b), individualizadas pelo espessamento da parede das fibras e pelo parênquima axial em linha ou faixas estreitas (Fig. 4e).

\section{Variações da anatomia do lenho entre as fisionomias}

Observa-se pela análise da Tabela 1 que dentre as dezesseis variáveis estudadas, oito apresentaram diferenças significativas do ponto de vista estatístico, sendo uma referente às fibras (comprimento), duas referentes aos raios parenquimáticos (altura e frequência) e cinco aos elementos de vaso (agrupamento: porcentagem de vasos solitários; comprimento, diâmetro, área e porcentagem). As árvores situadas em cerrado s.s. apresentaram fibras mais longas, raios mais baixos e em maior frequiência, bem como um menor número de vasos solitários, elementos de vaso mais curtos, com menor diâmetro tangencial médio (Tab. 1). Verifica-se ainda uma maior área ocupada por vasos e, consequentemente, maior porcentagem dos mesmos.

A presença de fibras mais longas em ambientes mésicos, amplamente relatada na literatura (Chalk 1989; Barajas-Morales 1985; Fahn 

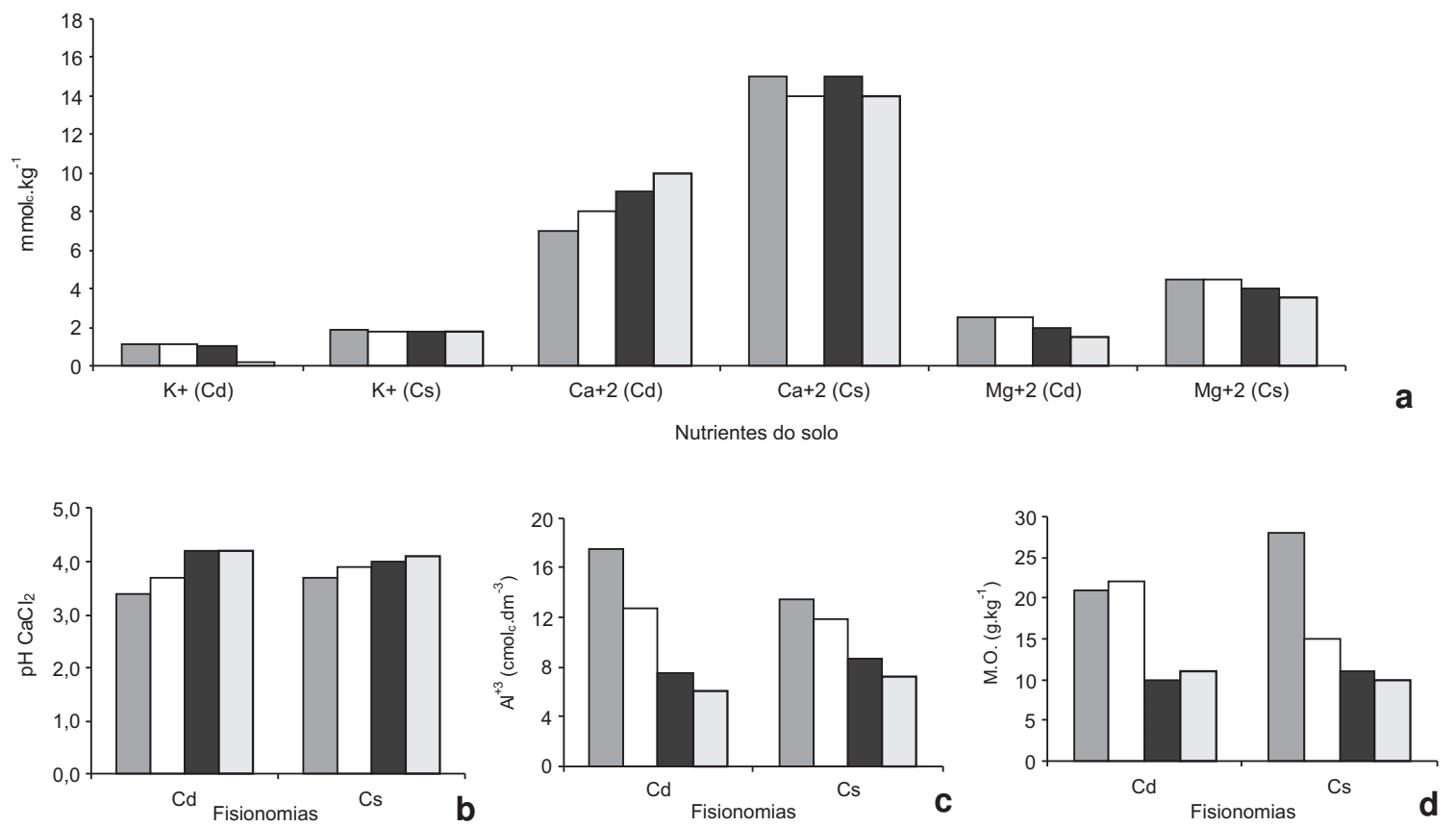

$$
\square 0-5 \mathrm{~cm} \quad \square 5-25 \mathrm{~cm} \quad \square 40-60 \mathrm{~cm} \quad \square 80-100 \mathrm{~cm}
$$

Figura 3 - Características dos solos das áreas de estudo nas fisionomias de cerradão (Cd) e cerrado sensu stricto (Cs) em diferentes profundidades. (A) Concentrações de potássio $\left(\mathrm{K}^{+}\right)$, cálcio $\left(\mathrm{Ca}^{+2}\right)$, magnésio $\left(\mathrm{Mg}^{+2}\right) \mathrm{em} \mathrm{mmol} \mathrm{kg}^{-1}$; (B) Valores de $\mathrm{pH}$ em $\mathrm{CaCl}_{2}$; (C) Concentração de alumínio trocável $\left(\mathrm{Al}^{+3}\right)$ em $\mathrm{cmol} \cdot \mathrm{dm}^{-3}$; (D) Matéria orgânica em g.kg $\mathrm{kg}^{-1}$. Fonte: Ruggiero et al., 2005.

Figure 3 - Soil characteristics for the studied areas in "cerradão" (Cd) and "cerrado sensu stricto" (Cs) at different dephts. (A) K',

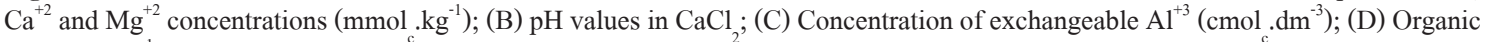
matter (g.kg $\left.{ }^{-1}\right)$. (Ruggiero et al. 2005).

et al. 1986; Alves \& Angyalossy-Alfonso 2002; Luchi 2004; Luchi et al. 2005), não foi observada neste caso em específico. Embora as áreas de estudo compartilhem das mesmas condições climáticas, um fator que as diferencia é a altitude, uma vez que o cerrado s.s. encontra-se em posição mais elevada (721-740 m) quando comparado ao cerradão (683$702 \mathrm{~m}$ ). Tendo-se em vista que as duas áreas se situam sobre neossolos quartzarênicos, há uma tendência de haver nas porções mais altas (cerrado s.s.) uma menor disponibilidade de água devido à drenagem excessiva que caracteriza essa classe de solo. Em contrapartida, a maior disponibilidade de nutrientes em cerrado s.s. (Fig. 3) pode ter promovido o desenvolvimento de fibras mais longas. Trabalhos realizados em ambiente de cerrado descrevem a presença de fibras mais longas em ambientes com maior disponibilidade hídrica (Luchi et al. 2005; Melo Júnior 2003), opondo-se ao encontrado para $C$. brasiliense neste estudo.
Quanto à relação entre desenvolvimento das fibras e teor de nutrientes em solos de cerrado, encontra-se na literatura relatos da presença de fibras mais longas para Xylopia aromatica (Lam.) Mart. em área com maiores teores de cálcio e magnésio (Luchi et al. 2005). Estes dados encontram-se em consonância com o obtido neste estudo, uma vez que os solos de cerrado s.s. apresentaram, comparativamente ao cerradão, teores mais elevados destes nutrientes (Fig.3). Tendo-se em vista a importância do cálcio como mensageiro secundário nas respostas das plantas a sinais ambientais e hormonais, bem como o papel do magnésio na ativação de enzimas envolvidas na respiração, fotossíntese e síntese de DNA e RNA (Taiz \& Zeiger 2004), seus maiores teores em área de cerrado s.s. podem ter influenciado na resposta das plantas às possíveis variações na disponibilidade hídrica, ainda que as diferenças nutricionais entre as áreas de estudo possam ser consideradas sutis. 

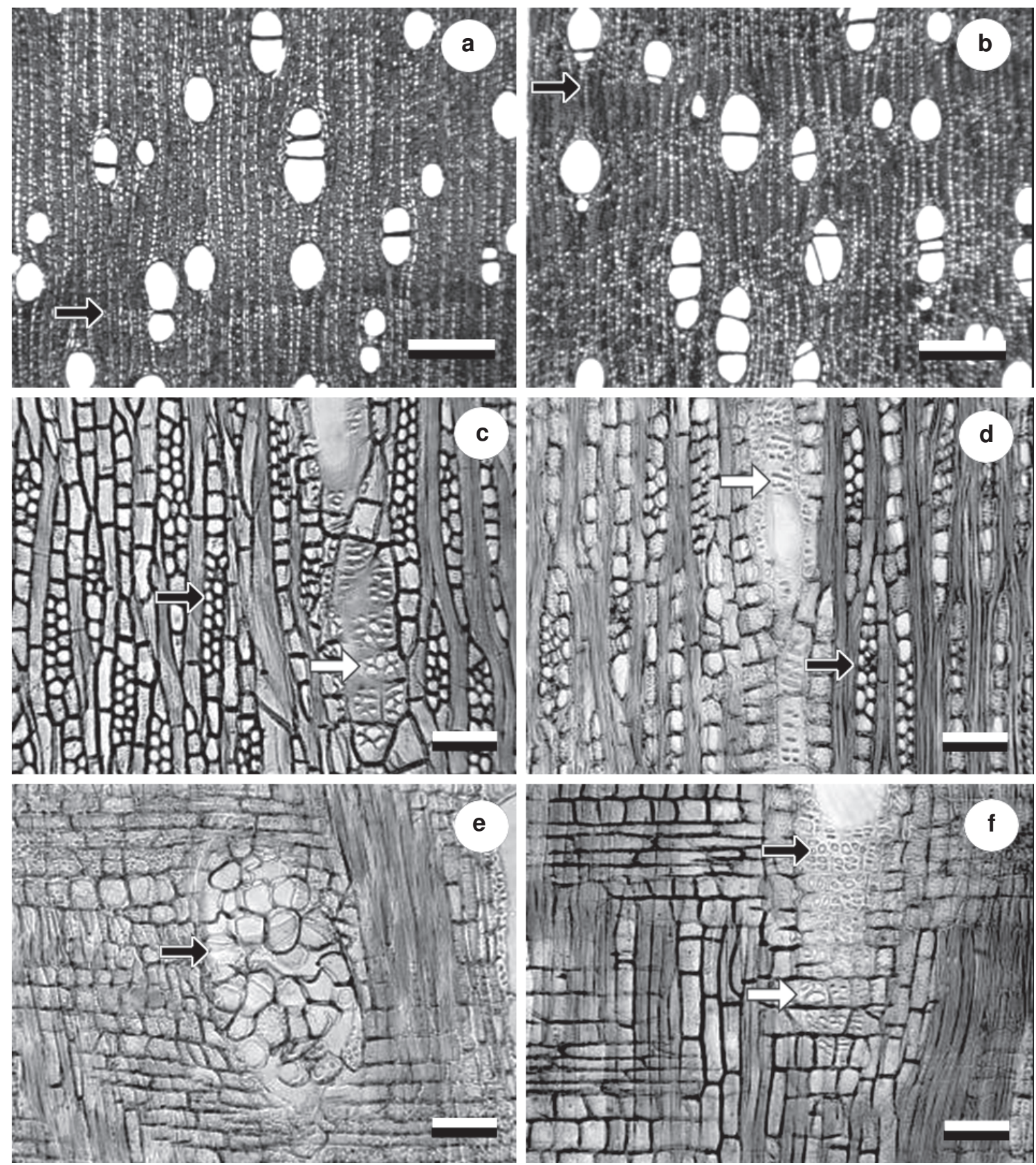

Figura 4 - Caryocar brasiliense nas fisionomias de cerradão (a, c, e) e cerrado sensu stricto (b, d, f). a-b. Seções transversais com destaque aos possíveis limites das camadas de crescimento; c-d. seções longitudinais tangenciais com indicações dos raios (setas escuras) e pontoações parênquimo-vasculares (setas claras); e-f. seção longitudinal radial indicando (e) intrusão de células parenquimáticas no lume de um elemento de vaso (seta escura), (f) pontoações radiovasculares semelhantes às parênquimovasculares. Escala barra: (a,b) $500 \mu \mathrm{m}$; (c-f) $100 \mu \mathrm{m}$.

Figure 4 - Caryocar brasiliense in "cerradão" (a, c, e) and "cerrado sensu stricto" (b, d, f). a-b. Cross sections showing limits of growth layers; c-d. tangential longitudinal sections showing rays (dark arrows) and intervessel pits (white arrows); e-f. radial longitudinal sections showing (e) intrusive parenchyma cells into vessel lumen (dark arrow), (F) ray-vessel pits similar to axial parenchyma-vessel pits. Scale bar: (a,b) $500 \mu \mathrm{m}$; (c-f) $100 \mu \mathrm{m}$. 
Tabela 1 - Valores médios e desvios-padrão (entre parênteses) dos caracteres anatômicas do xilema secundário em cada população de $C$. brasiliense no cerradão e cerrado sensu stricto.

Table 1 - Mean values and standard deviation (between brackets) of wood anatomical features analyzed in each population of C. brasiliense in "cerradão" and "cerrado" sensu stricto.

\begin{tabular}{|c|c|c|c|c|}
\hline $\begin{array}{l}\text { Elementos } \\
\text { anatômicos }\end{array}$ & $\begin{array}{l}\text { Variáveis } \\
\text { analisadas }\end{array}$ & & Cerradão & $\begin{array}{l}\text { Cerrado } \\
\text { sensu stricto }\end{array}$ \\
\hline \multirow[t]{3}{*}{ Fibras } & Comprimento $(\mu \mathrm{m})$ & & $2085,05(282,24) b^{*}$ & $2151,99(270,91) a^{*}$ \\
\hline & Diâmetro da fibra $(\mu \mathrm{m})$ & & $23,97(4,64) \mathrm{a}$ & $23,64(4,13) a^{*}$ \\
\hline & Espessura da parede $(\mu \mathrm{m})$ & & $10,52(2,37) a^{*}$ & $10,72(2,02) a^{*}$ \\
\hline \multirow[t]{3}{*}{ Raios parenquimáticos } & Altura $(\mu \mathrm{m})$ & & $460,97(138,26) a^{*}$ & $427,87(137,18) b^{*}$ \\
\hline & Largura $(\mu \mathrm{m})$ & & $38,23(8,84) a^{*}$ & $38,28(9,74) a^{*}$ \\
\hline & $\begin{array}{l}\text { Frequência de raios } \\
\left(\text { raios } / \mathrm{mm}_{\text {linear }}\right)\end{array}$ & & $22,84(2,43) b^{*}$ & $24,38(1,50) a^{*}$ \\
\hline \multirow[t]{10}{*}{ Vasos } & $\begin{array}{l}\text { Porcentagem de vasos } \\
\text { solitários e múltiplos }\end{array}$ & $\mathrm{S}$ & $28,27 a^{*}$ & $23,04 b^{*}$ \\
\hline & & $\mathrm{M}_{2}$ & $36,03 \mathrm{a}^{*}$ & $37,10 \mathrm{a}^{*}$ \\
\hline & & $\mathrm{M}_{3}$ & $24,31 \mathrm{a}^{*}$ & $23,84 a^{*}$ \\
\hline & & $\mathrm{M}_{4}$ & $7,80 \mathrm{a}^{*}$ & $12,05 \mathrm{a}^{*}$ \\
\hline & & $M \geq_{5}$ & $3,60 \mathrm{a}^{*}$ & $3,97 \mathrm{a}^{*}$ \\
\hline & $\begin{array}{l}\text { Comprimento dos } \\
\text { elementos de vaso }(\mu \mathrm{m})\end{array}$ & & $512,25(132,96) \mathrm{a}^{*}$ & $483,03(114,20) b^{*}$ \\
\hline & Diâmetro dos vasos $(\mu \mathrm{m})$ & & $144,52(55,32) b^{*}$ & $156,51(56,89) \mathrm{a}^{*}$ \\
\hline & $\begin{array}{l}\text { Frequência de vasos } \\
\left(\text { vasos } / \mathrm{mm}^{2}\right)\end{array}$ & & $6,84(1,93) a^{*}$ & $6,79(2,05) a^{*}$ \\
\hline & Área vasos $\left(\mathrm{mm}^{2} / \mathrm{mm}^{2}\right)$ & & $0,1201(0,0261) b^{*}$ & $0,1355(0,0270) a^{*}$ \\
\hline & Porcentagem de vasos & & $12,10(2,61) b^{*}$ & $13,65(2,73) a^{*}$ \\
\hline
\end{tabular}

Médias seguidas da mesma letra não diferem entre si; asteriscos indicam diferenças significativas entre árvores da mesma localidade (teste de Tuckey, $\alpha 0,05$ ).

Raios mais altos e menos frequentes foram encontrados na fisionomia de cerradão, área com a menor disponibilidade de nutrientes (Ruggiero \& Pivello 2005; Ruggiero et al. 2006). Dados semelhantes foram obtidos para altura de raios por Melo Júnior (2003) e Luchi et al. (2005) em áreas de cerrado no Paraná e em São Paulo, respectivamente. Atribui-se ao parênquima radial a função de transporte de íons entre o xilema e o floema (Läuchli 1972), bem como a função de tecido acessório aos elementos condutores, juntamente com o parênquima axial, promovendo o aumento do fluxo nos vasos através da mobilização de substâncias osmoticamente ativas (Braun 1984). Com isso, raios com maiores dimensões podem auxiliar no transporte de água e nutrientes nas plantas que os possuem, tornando-as mais adaptadas a condições ambientais tidas como desfavoráveis, como observado para o cerradão.
As árvores presentes em área de cerradão também apresentaram maior porcentagem de vasos solitários, elementos de vaso mais longos, com menor diâmetro, bem como com menor área de condução. Vasos múltiplos conferem uma maior segurança na condução hidráulica, uma vez que a resistência à cavitação é dada pela relação entre (i) espessura da parede do vaso e vaso adjacente (no caso de vasos múltiplos) e (ii) diâmetro do lume (Jacobsen et al. 2005). A relação exposta reforça a tese de que vasos múltiplos conferem maior segurança no transporte, além de proporcionarem uma rota alternativa em casos de embolismos, mais propensos a ocorrer em regiões com estações/clima seco (Alves \& Angyalossy-Alfonso 2000). A maior porcentagem de vasos solitários e a presença de elementos de vaso mais longos em área de cerradão confirmam a tendência de que estes são mais frequentes em ambientes de maior disponibilidade hídrica. 
Elementos de vasos mais longos e largos foram relatados em áreas de cerrado para ambientes mésicos (Melo Júnior 2003) e em ambientes de floresta em relação ao cerrado (Pereira 1990). A presença de elementos axiais mais longos em condições de maior disponibilidade hídrica se deve ao maior turgor celular à que as iniciais fusiformes estão submetidas nestes ambientes (Levitt 1972). De modo geral, árvores de áreas mais secas possuem elementos de vaso de menor diâmetro (Baas et al. 1983; Baas \& Carlquist 1985; Luchi 2004) e em maior frequência, como forma de garantir o fluxo hídrico (BarajasMorales 1985; Lindorf 1994; Alves \& AngyalossyAlfonso 2000). Para C. brasiliense, a frequência não diferiu significativamente entre as fisionomias. No entanto, a presença de vasos de menor diâmetro em ambiente de maior disponibilidade hídrica (cerradão) contrariou as tendências descritas pelos autores supracitados, mas se assemelha ao encontrado por Mina-Rodrigues (1986), Ceccantini (1996) e Marcati et al. (2001), que encontraram vasos de maior diâmetro em áreas de cerrado/cerradão em relação à floresta.

Sabe-se que as dimensões das células vegetais não dependem apenas de fatores ambientais, mas também das suas potencialidades genéticas e características ontogenéticas. As células derivadas do câmbio se expandem longitudinalmente e radialmente até atingir seu tamanho final durante a formação da parede primária (Plomion et al. 2001). Durante esse período, se houver a manutenção da pressão de turgor dessas células em diferenciação mediante o estímulo auxínico adequado, os elementos crescerão tanto em diâmetro como em comprimento (Carlquist 1988; Levitt 1972). Neste aspecto, a presença concomitante de fibras mais longas e elementos de vaso mais curtos com maior diâmetro tangencial em ambiente de cerrado s.s. é um ponto que carece de melhores elucidações, visto que ambos elementos derivam de células iniciais fusiformes e foram formados durante o mesmo período de crescimento da árvore. Uma vez que o nível de auxina livre nas células parece influenciar na freqüência e nas dimensões dos elementos de vaso (Taiz \& Zeiger 2004), essas células podem ter apresentado uma resposta mais significativa às variações ambientais quando comparados às fibras. Há ainda que se considerar a variação entre árvores, uma vez que foram constatadas diferenças significativas entre metade dos indivíduos dentro da fisionomia de cerradão, sendo o comprimento dos elementos de vasos especialmente heterogêneo no referido ambiente. Já em cerrado s.s., as árvores apresentaram maior homogeneidade dessas variáveis, diferindo de forma mais significativa das árvores de cerradão do que dentro da mesma fisionomia.

\section{Variações anatômica do lenho em cada fisionomia}

A análise de variância indicou para todas as variáveis analisadas diferenças significativas dentro das áreas de estudo, excetuando-se apenas o diâmetro das fibras para as árvores de cerradão (Tab. 1). Nota-se, com isso, uma grande variação entre os indivíduos mesmo quando utilizados os valores médios por árvore, fato corroborado pela análise de agrupamentos (Fig. 5). O maior agrupamento formado exclusivamente por árvores de uma mesma fisionomia consiste em três indivíduos de cerrado s.s. (Cs09, Cs11 e Cs12), não ocorrendo o mesmo para a fisionomia de cerradão, localidade em que as árvores são mais heterogêneas. Nota-se ainda semelhança entre árvores de cerrado s.s. (Cs08 e Cs10) e árvores de cerradão, diferindo das demais na mesma fisionomia (Cs07, Cs09, Cs11, Cs12). De maneira análoga, os indivíduos Cd04, $\mathrm{Cd} 05$ e Cd06 demonstram possuir maior proximidade com árvores de cerrado s.s. quando comparadas aos indivíduos da mesma fisionomia (cerradão).

A correlação entre variáveis obtida pela análise multivariada de componentes principais (PCA) (Tab. 2) demonstrou a existência de uma forte correlação positiva entre área e porcentagem da área ocupada por vasos $(0,9922)$, uma vez que representam a mesma variável em unidades distintas. Em relação às fibras, o comprimento apresentou correlação negativa com a espessura da parede $(-0,4102)$ e de forma menos pronunciada

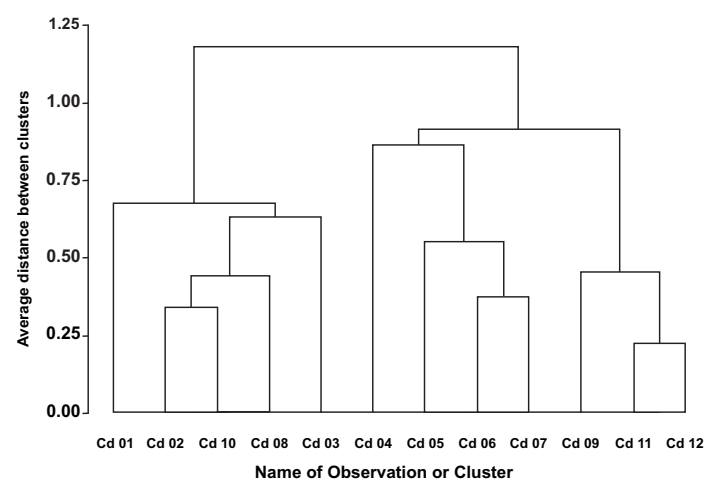

Figura 5 - Dendrograma resultante da análise de agrupamentos demonstrando similaridades entre as árvores. Árvores Cd01 a Cd06: cerradão; árvores Cs07 a Cs12: cerrado sensu stricto.

Figure 5-Dendrogram resulting from Cluster Analysis showing similarities between trees. Trees Cd01 to Cd06: "cerradão"; trees Cs07 to Cs12: “cerrado" sensu stricto. 
Tabela 2 - Matriz de correlação entre variáveis anatômicas obtidas pela análise multivariada de componentes principais. Table 2 - Correlation between wood anatomical features of all individuals of the two populations generated by a Principal Component Analysis.

\begin{tabular}{|c|c|c|c|c|c|c|c|c|c|c|c|}
\hline & Cf & Df & EPf & Ar & $\mathbf{L r}$ & Dv & $\mathrm{Cv}$ & Fr & Fv & Av & $\mathbf{P v}$ \\
\hline Cf & 1,0000 & & & & & & & & & & \\
\hline Df & $-0,3998$ & 1,0000 & & & & & & & & & \\
\hline EPf & $-0,4102$ & 0,7336 & 1,0000 & & & & & & & & \\
\hline $\mathbf{A r}$ & 0,1315 & $-0,1219$ & $-0,4703$ & 1,0000 & & & & & & & \\
\hline $\mathbf{L r}$ & $-0,5495$ & 0,1014 & 0,2302 & $-0,3002$ & 1,0000 & & & & & & \\
\hline Dv & 0,1961 & $-0,3891$ & $-0,3108$ & $-0,5781$ & 0,4219 & 1,0000 & & & & & \\
\hline $\mathrm{Cv}$ & 0,4215 & $-0,1590$ & $-0,3809$ & 0,4305 & $-0,4740$ & $-0,2900$ & 1,0000 & & & & \\
\hline Fr & 0,3475 & $-0,2138$ & 0,0293 & 0,0778 & $-0,4199$ & $-0,1605$ & 0,0514 & 1,0000 & & & \\
\hline Fv & 0,3367 & 0,2055 & $-0,0241$ & 0,4327 & $-0,3935$ & $-0,3821$ & 0,0927 & $-0,0664$ & 1,0000 & & \\
\hline Av & 0,4708 & $-0,1365$ & $-0,1707$ & $-0,2378$ & $-0,0039$ & 0,5417 & $-0,0482$ & $-0,1397$ & 0,3888 & 1,0000 & \\
\hline $\mathbf{P v}$ & 0,4726 & $-0,1799$ & $-0,2060$ & $-0,1962$ & $-0,0098$ & 0,5200 & $-0,0755$ & $-0,1274$ & 0,4431 & 0,9922 & 1,0000 \\
\hline
\end{tabular}

(Cf) comprimento das fibras em $\mu \mathrm{m}$; (Df) diâmetro das fibras em $\mu \mathrm{m}$; (EPf) espessura da parede das fibras em $\mu \mathrm{m}$; (Ar) altura dos raios em $\mu \mathrm{m}$; (Lr) largura dos raios em $\mu \mathrm{m}$; (Dv) diâmetro dos vasos em $\mu \mathrm{m}$; (Cv) comprimento de vasos em $\mu \mathrm{m}$; (Fr) frequência de raios por mm ${ }^{2}$; (Fv) frequência de vasos por mm ${ }^{2}$; (Av) área de vasos: $\mathrm{mm}^{2} \mathrm{~mm}^{-2} ;(\mathrm{Pv})$ porcentagem de vasos.

com o diâmetro $(-0,3998)$, no entanto, não se observou correlação negativa com o comprimento dos elementos de vaso $(0,4215)$ como indicado pela análise de variância. Esse aspecto leva a crer que o fato da análise de variância indicar tendências opostas para os comprimentos das fibras e elementos de vaso decorre de uma significativa variabilidade entre árvores. Embora seja esperada a existência de uma relação direta entre comprimento e diâmetro dos elementos de vaso, ela ocorreu de modo inverso neste estudo, ainda que de forma pouco expressiva $(-0,2900)$. A espessura da parede das fibras se correlacionou positivamente com o diâmetro das mesmas $(0,7336)$, assim como diâmetro e área de vasos $(0,5417)$ e diâmetro e porcentagem da área ocupada pelos mesmos $(0,5200)$. Constatouse a presença de correlações negativas entre as dimensões longitudinais (comprimento/altura) e radiais (diâmetro/largura) dos elementos anatômicos, como observado para altura de raios e diâmetro de vasos $(-0,5781)$, e para largura de raios e comprimento das fibras (-0,5495). Essas correlações podem indicar que há um favorecimento do crescimento de uma dimensão em detrimento da outra, ou seja, o maior crescimento longitudinal pode implicar em um menor crescimento radial e vice-versa. Ainda que essas correlações tenham sido pouco expressivas, essa tendência geral pode ser verificada para comprimento e diâmetro das fibras $(-0,3998)$, comprimento e diâmetro dos vasos $(-0,2900)$ e altura e largura de raios $(-0,3002)$.
A análise anatômica do lenho indicou a presença de diferenças estruturais entre as árvores nas áreas de estudo, no entanto, para grande parte das variáveis, as respostas não ocorreram em consonância com as tendências amplamente relatadas para as floras mundiais (Chalk 1989; BarajasMorales 1985; Fahn et al. 1986; Alves \& Angyalossy-Alfonso 2002). Essa discrepância residiu principalmente na presença de fibras mais curtas, elementos de vaso mais estreitos e menor área de condução de água para a localidade com maior disponibilidade hídrica, no caso, a fisionomia de cerradão. Entretanto, a presença de raios mais altos na área com menor disponibilidade de nutrientes (cerradão) está em concordância com o disposto na literatura. A variabilidade significativa entre árvores, principalmente dentro de uma mesma área de estudo, indicou que a diferenciação das fisionomias ocorre de forma gradual, não se observando um conjunto de características anatômicas que tipifiquem indivíduos das duas fisionomias em função das variáveis ambientais. Esse aspecto pôde ser observado na análise de agrupamentos, indicando em alguns casos uma maior semelhança entre árvores procedentes de fisionomias distintas do que dentro da mesma área de estudo. A existência de uma grande variação de resultados obtidos em estudos anatômicos de espécies do Cerrado indica que pouco se sabe sobre a dinâmica de crescimento das árvores nestes ambientes, bem como suas estratégias adaptativas. Deste modo, dada a complexidade das 
interações entre os fatores internos e externos que atuam na diferenciação e desenvolvimento das células do xilema, bem como a impossibilidade de analisá-los separadamente, torna-se desejável a ampliação da amostragem para um maior número de áreas de estudo, sendo estas selecionadas de modo a abranger uma maior variação ambiental na localidade em questão.

\section{Agradecimentos}

À CAPES, a concessão da bolsa de estudos de mestrado à primeira autora; ao IPEF, o financiamento das viagens de campo. Ao IF-SP, em especial ao Sr. Heverton José Ribeiro, por autorizar a realização do trabalho no Parque Estadual da Vassununga. À Prof. Dra. Graciela Inez Bolzón Muñiz do Departamento de Ciências Florestais da UFPR, o apoio, incentivo e por disponibilizar a infraestrutura necessária para a realização do trabalho. À técnica do Laboratório de Anatomia e Identificação de Madeiras da ESALQ/USP, Maria Aparecida R.C. Bermudez, a ajuda incondicional e empenho na confecção dos cortes histológicos e demais atividades laboratoriais. Aos docentes da ESALQ/USP, Dr. João Luiz Ferreira Batista e Dr. Carlos Tadeu dos Santos Dias, o direcionamento e auxílio nas análises estatísticas.

\section{Referências}

Alves, E.S. \& Angyalossy-Alfonso, V. 2000. Ecological trends in the wood anatomy of some Brazilian species. 1. Growth rings and vessels. Iawa Journal 20: 3-30.

Alves, E.S. \& Angyalossy-Alfonso, V. 2002. Ecological trends in the wood anatomy of some Brazilian species. 2. Axial parenchyma, rays and fibres. Iawa Journal 23: 391-418.

Alvim, P.T. \& Araújo, W.A. 1952. El suelo como factor ecológico en el desarrolo de la vegetación en el centrooeste del Brasil. Turrialba 2: 153-160.

Araujo, P.A.M. \& Mattos, A. 1973. Estrutura das madeiras de Caryocaraceae. Arquivos do Jardim Botânico do Rio de Janeiro 19: 5-47.

Arens, K. 1958a. Considerações sobre as causas do xeromorfismo foliar. Separata de Boletim Botânica 224: 23-56.

Arens, K. 1958b. O cerrado como vegetação oligotrófica. Separata de Boletim Botânica 224: 57-77.

Baas, P. 1973. The wood anatomical range in Ilex (Aquifoliaceae) and its ecological and phylogenetic significance. Blumea 21: 193-258.

Baas, P. \& Carlquist, S. 1985. A comparison of the ecological wood anatomy of the floras of Southern California and Israel. Iawa Bulletin 6: 141-159.
Baas, P. \& Schweingruber, F.H. 1987. Ecological trends in the wood anatomy of trees, schrubs and climbers from Europe. Iawa Bulletin 8: 245-274.

Baas, P; Werker, E.E. \& Fahn, A. 1983. Some ecological trends in vessel characters. Iawa Bulletin 4: 141-159.

Barajas-Morales, J. 1985. Wood structural differences between trees of two tropical forests in Mexico. Iawa Bulletin 6: 355-364.

Braun, H.J. 1984. The significance of accessory tissues of the hydrosystem for osmotic water shifting as the second principle of water ascent, with some thoughts concerning the evolution of trees. Iawa Bulletin 5: 275-294.

Carlquist, S. 1975. Ecological strategies in xylem evolution. University of California Press, Berkeley. 259p.

Carlquist, S. 1988. Comparative wood anatomy: systematic, ecological and evolutionary aspects of dicotyledon wood. Springer-Verlag, Berlin. 436p.

Carlquist, S. \& Hoekman, D.A. 1985. Ecological wood anatomy of woody Southern Californian flora. Iawa Bulletin 6: 319-347.

Ceccantini, G.C.T. 1996. Anatomia ecológica do lenho de espécies de cerrado e mata: Casearia sylvestris Sw. e Machaerium villosum Vog. Dissertação de Mestrado. Universidade de São Paulo, São Paulo. 117p.

Chalk, L. 1989. The effect of ecology conditions on wood anatomy. In: Metcalfe, C.R. \& Chalk, L. Anatomy of the dicotyledons. Vol. 2. Oxford Science, Oxford. Pp. 126-152.

Comision Panamericana de Normas Técnicas - COPANT. 1974. Método para la descripción de las características generales, macroscópicas y microscópicas de la madera angiospermas dicotiledóneas 30: 1-19.

Coutinho, L.M. 2008. Cerrado. Disponível em <http:// eco.ib.usp.br/cerrado/>. Acesso em 20 fevereiro 2008.

Dickison, W. C. 2000. Integrative plant anatomy. Harcourt Academic Press, San Diego. 533p.

Fahn, A.; Werker, E. \& Baas, P. 1986. Wood anatomy and identification of trees and shrubs from Israel and Adjacent Regions. The Israel Academy of Sciences and Humanities, Jerusalem. 221p.

Franco, A.C. \& Lüttge, U. 2002. Midday depression in savanna trees: coordinated adjustments in photochemical, efficiency, photorespiration, $\mathrm{CO}_{2}$ assimilation and water use efficieny. Oecologia 131: 356-365.

Haridasan, M. 2005. Competição por nutrientes em espécies arbóreas do cerrado. In: Scariot, A.; SousaSilva, J. C. \& Felfili, J. M. (org.). Cerrado: ecologia, biodiversidade e conservação. Ministério do Meio Ambiente, Brasília. Pp. 167-178.

IAWA Committee. 1989. Iawa list of microscopic features for hardwood identification. IAWA Bulletin 10: 219-332.

Instituto Brasileiro do Meio Ambiente e dos Recursos Naturais Renováveis - IBAMA. 1992. Normas de procedimentos em estudos de anatomia da madeira: I Angiospermae. II Gimnospermae. Diretoria de 
incentivo à pesquisa e divulgação/Laboratório de Produtos Florestais, Brasília.

Jacobsen, A.L.; Ewers, F.W.; Pratt, R.B.; Paddock III, W.A. \& Davis, S.D. 2005. Do xylem fibers affect Vessel Cavitation Resistance? Plant Physiology 139: 546-556.

Köppen, W. 1948. Climatologia. Fondo de Cultura Económica, México. 479p.

Läuchli, A. 1972. Translocation of organic solutes. Annual Review Plant Physiology and Plant Molecular Biology 23: 197-218.

Levitt, J. 1972. Responses of plants to environmental stresses. Academic Press, New York. 697p.

Lindorf, H. 1994. Eco-anatomical wood features of species from a very dry tropical forest. Iawa Journal 15: 361-376.

Luchi, A.E. 2004. Anatomia do lenho de Croton urucurana Baill. (Euphorbiaceae) de solos com diferentes níveis de umidade. Revista Brasileira de Botânica 27: 271-280.

Luchi, A.E.; Silva, L.C.P. \& Moraes, M.A. 2005. Anatomia comparada do lenho de Xylopia aromatica (Lam.) Mart. em áreas de cerrado e de plantação de Pinus elliottii Engelm. Revista Brasileira de Botânica 28: 809-820.

Marcati, C.R.; Angyalossy-Alfonso, V. \& Benetati, L. 2001. Anatomia comparada do lenho de Copaifera langsdorffii Desf. (Leguminosae-Caesalpinoideae) de floresta e cerradão. Revista Brasileira de Botânica 24: 311-320.

Mello, E.C. 1970. Estudo anatômico das madeiras do gênero Caryocar Linn. Brasil Florestal 1: 54-62.

Melo Júnior, J.C.F. 2003. Anatomia ecológica da folha e do lenho de Copaifera langsdorffii Desf. (Leguminosae) do Parque Estadual do Cerrado, Jaguariaíva - PR. Dissertação de Mestrado. Universidade Federal do Paraná, Curitiba. 73p.

Mina-Rodriges, E.M.C. 1986. Estudo morfo-anatômico dos órgãos vegetativos de Pera glabrata Baill. (Euphorbiaceae), em material procedente de mata mesófila semidecídua e de cerrado. Dissertação de Mestrado. Universidade Estadual Paulista, Rio Claro. 103p.

Naves-Barbiero, C.C.; Franco, A.C.; Bucci, S.J. \& Goldstein, G. 2000. Fluxo de seiva e condutância estomática de duas espécies lenhosas sempre-verdes no campo sujo e cerradão. Revista Brasileira de Fisiologia Vegetal 12: 119-134.

Pereira, B.A.S. 1990. Estudo morfo-anatômico da madeira, casca e folha de duas variedades vicariantes de Sclerolobium paniculatum de mata e cerrado. Dissertação de Mestrado. Escola Superior de Agricultura Luiz de Queiroz, Universidade de São Paulo, Piracicaba. 209p.
Perez, S.C.J.G.A \& Moraes, J.A.P.V. 1991. Determinação do potencial hídrico, condutância estomática e potencial osmótico em espécies dos estratos arbóreos, arbustivo e herbáceo de um cerradão. Revista Brasileira de Fisiologia Vegetal 3: 27-37.

Plomion, C; Leprovost, G. \& Stokes, A. 2001. Wood formation in trees. Plant Physiology 127: 1513-1523.

Ratter, J.A.S.; Bridgewater, S., Atkins, R. \& Ribeiro, J.F. 1996. Analysis of floristic composition of the Brazilian cerrado vegetation. II. Comparison of the woody vegetation of 98 areas. Edinburgh Journal of Botany 53: 153-180.

Rawitscher, F. \& Ferri, M.G. 1943. Profundidade dos solos e vegetação em campos cerrados do Brasil meridional. Anais da Academia Brasileira de Ciências 15: 267-298.

Ribeiro, J.F. \& Walter, B.M.T. 1998. Fitofisionomias do bioma Cerrado. In: Sano, S.M. \& Almeida, S.P. Cerrado: ambiente e flora. EMBRAPA-CPAC, Planaltina. Pp. 89-152.

Rizzini, C.T. 1976. Tratado de fitogeografia do Brasil: aspectos ecológicos. Hucitec, São Paulo. 327p.

Rolim, G.S. \& Sentelhas, P. C. 2008. Balanços hídricos climatológicos de quinhentas localidades brasileiras. Disponível em <http://ce.esalq.usp.br/departamentos/ lce/bhbrasil/Saopaulo>. Acesso em 20 julho 2008.

Ruggiero, P.G.C.; Batalha, M.A.; Pivello, V.R. \& Meirelles, S.T. 2002. Vegetation-soil relationships in cerrado (Brazilian savanna) and semideciduous forest, Southeastern Brazil. Plant Ecology 160: 1-16.

Ruggiero, P.G.C. \& Pivello, V.R. 2005. O solo e a comunidade vegetal. In: Pivello, V.R. \& Varanda, E.M. (org.). O cerrado Pé-de-Gigante (Parque Estadual da Vassununga, São Paulo) - ecologia e conservação. Secretaria de Estado do Meio Ambiente, São Paulo. Pp. 173-188.

Ruggiero, P.G.C.; Pivello, V.R.; Spavorek, G.; Teramoto, E. \& Pires Neto, A.G. 2006. Relação entre solo, vegetação e topografia em área de cerrado (Parque Estadual de Vassununga, SP): como se expressa em mapeamentos? Acta Botanica Brasilica 20: 383-394.

Silva Júnior, M.C. 2005. Guia de campo: 100 árvores do cerrado. Ed. Rede de Sementes do Cerrado, Brasília. $278 \mathrm{p}$.

Steel, R.G.D.; Torrie, J.H. \& Dickey, D.A. 1997. Principles and procedures of statistics: a biometrical approach. McGraw Hill Book, New York. 666p.

Taiz, L. \& Zeiger, E. 2004. Fisiologia vegetal. Artmed, Porto Alegre. 690p.

Warming, E. 1908. Lagoa Santa: contribuição para a geographia phytobiologica. Imprensa Oficial do Estado de Minas Gerais, Bello Horizonte. 
